\title{
BMJ Open CoMET: a protocol for a randomised controlled trial of co-commencement of METformin as an adjunctive treatment to attenuate weight gain and metabolic syndrome in patients with schizophrenia newly commenced on clozapine
}

To cite: Siskind D, Friend N, Russell A, et al. CoMET: a protocol for a randomised controlled trial of $\mathrm{co}^{-}$ commencement of METformin as an adjunctive treatment to attenuate weight gain and metabolic syndrome in patients with schizophrenia newly commenced on clozapine. BMJ Open 2018:8:e021000. doi:10.1136/ bmjopen-2017-021000

- Prepublication history and additional material for this paper are available online. To view these files, please visit the journal online (http://dx.doi. org/10.1136/bmjopen-2017021000).

Received 10 December 2017 Revised 19 January 2018 Accepted 29 January 2018

Check for updates

For numbered affiliations see end of article.

Correspondence to

Dr Dan Siskind;

d.siskind@uq.edu.au

\section{ABSTRACT}

Introduction Clozapine, while effective in treatment refractory schizophrenia, is associated with significant weight gain, heart disease and increased risk of type 2 diabetes mellitus (T2DM). Although there is evidence for weight loss with metformin for people with obesity who are already taking clozapine, there have been no published trials that have investigated the effect of metformin in attenuating weight gain at the time of clozapine initiation. Methods and analysis A 24-week double-blind placebocontrolled trial of concomitant prescription of metformin at clozapine commencement. Eighty-six people being commenced on clozapine will be randomised to placebo or metformin (variable dose, up to $2 \mathrm{~g} /$ day). The primary outcome is comparative end point body weight, between the placebo and metformin groups. Secondary outcomes are comparative rates of conversion to T2DM, alteration of metabolic syndrome parameters, proportion gaining $>5 \%$ body weight and changes in diet and appetite. We will additionally examine biomarkers associated with change in weight among trial participants.

Ethics and dissemination Ethics approval was granted by the Metro South Human Research Ethics Committee HREC/17/QPAH/538-SSA/17/QPAH/565. We plan to submit a manuscript of the results to a peer-reviewed journal, and present results at conferences, consumer forums and hospital grand rounds.

Trial registration number ACTRN12617001547336; Preresults.

\section{INTRODUCTION}

Schizophrenia is associated with substantial disability and excess morbidity/mortality; life expectancy is curtailed by over 16 years ${ }^{1}$ with over a third of excess deaths attributable to

\section{Strengths and limitations of this study}

- This is the first randomised controlled trial investigating metformin for amelioration of clozapine associated weight gain at the time of clozapine initiation.

- If effective, co-commencement of metformin at the time of clozapine initiation could reduce the cardiovascular and metabolic disease burden of clozapine.

- People with treatment refractory schizophrenia being commenced on clozapine will be a challenging group from which to recruit.

- Dropouts from the trial may occur when people commenced on clozapine cease clozapine.

cardiovascular disease and type 2 diabetes mellitus (T2DM). ${ }^{1}$ Increased risk of cardiometabolic disease in this population is multifactorial with possible contributing components including genetic predisposition to developing T2DM, ${ }^{2}$ reduced physical activity, ${ }^{3}$ suboptimal nutrition ${ }^{4}$ and glucose dysregulation associated with antipsychotic medications. ${ }^{5}$

Although other antipsychotic medications are effective treatments for schizophrenia, ${ }^{6}$ approximately $20 \%-33 \%$ of patients remain treatment refractory. ${ }^{7}$ Treatment refractory schizophrenia is defined as non-response with ongoing psychotic symptoms and functional deficits despite adequate trials of at least two different antipsychotic medications. ${ }^{8}$ For people with treatment refractory 
schizophrenia, clozapine is the most effective medication for reducing the positive symptoms of schizophrenia, ${ }^{9}$ and the rate of psychiatric hospitalisations. ${ }^{10}$ Compared with other antipsychotic medications, clozapine is associated with the highest rates of weight gain, T2DM and metabolic syndrome. ${ }^{5}$ A representative survey of people with schizophrenia in Australia found that, compared with people on other antipsychotic medications, people on clozapine were almost twice as likely to develop T2DM, and more than twice as likely to develop metabolic syndrome. ${ }^{11}$ In an American study of clozapine users with a 10-year follow-up, $43 \%$ of participants developed T2DM. The mean weight gain was $13.5 \mathrm{~kg}$, of which $4.5 \mathrm{~kg}$ occurred in the first 10 weeks of commencing clozapine. ${ }^{2}$

Weight gain is a significant concern for patients. It is associated with poorer quality of life outcomes, ${ }^{13}$ creates barriers to social engagement ${ }^{14}$ and is the most distressing side effect reported to callers of mental health helplines. ${ }^{15}$ Weight gain also reinforces patients' negative views of themselves and may compromise adherence with treatment. ${ }^{15}$ Furthermore, there is an established body of evidence that being overweight or obese increases the risk of all-cause mortality with higher weight associated with higher mortality risk. ${ }^{16} 17$

Although there is some evidence for the efficacy of lifestyle modification interventions for people with schizophrenia, ${ }^{3}$ poor rates of uptake of lifestyle modification remain a barrier to their effectiveness. ${ }^{3}$ Cognitive deficits associated with schizophrenia can contribute to difficulties with meal planning and accessing physical activity programmes. ${ }^{18}$ Consequently, interest is increasing in effectiveness and acceptability of other interventions such as oral medication.

Among people taking clozapine who are obese, there is increasing evidence that metformin can lead to modest weight loss. ${ }^{19}$ Metformin, a biguanide antihyperglycaemic commonly used in the management of $\mathrm{T} 2 \mathrm{DM},{ }^{20}$ reduces fasting glucose and triglyceride (TG) and high-density lipoprotein (HDL) cholesterol. $^{21}$ Antihyperglycaemic properties are attributed primarily to suppression of hepatic gluconeogenesis and increased peripheral insulin sensitivity. ${ }^{20}$ In people without T2DM who are not on antipsychotic medications, metformin can lead to mild weight loss. ${ }^{22}$ Furthermore, when initiated in overweight patients with newly diagnosed T2DM, metformin can reduce the long-term risk of any T2DM end point and all-cause mortality. ${ }^{23}$ Metformin also has a much lower rate of hypoglycaemia compared with other antidiabetic drugs such as sulfonamides. ${ }^{24}$

There is also evidence that metformin increases the production of glucagon-like peptide (GLP-1), an intestinal epithelium produced peptide following food consumption. ${ }^{25}$ In turn, GLP-1 stimulates insulin secretion while inhibiting glucagon secretion, and also appears to regulate appetite by inducing satiety. ${ }^{26}$ Metformin's role in GLP-1 regulation is of particular relevance for people on clozapine as clozapine disrupts the GLP-1 pathway in the intestinal epithelium, thereby reducing GLP-1 levels. ${ }^{27}$
As such, it is possible that metformin may have particular benefits with respect to weight gain associated with clozapine (vs other antipsychotics).

A recent meta-analysis by our group demonstrated that addition of metformin contributed to weight loss of $>3 \mathrm{~kg}$ among people already taking clozapine who are obese, ${ }^{19}$ with significant improvements in body mass index (BMI), and on three out of the five components of the metabolic syndrome: waist circumference, fasting glucose and triglycerides.

There is, however, an absence of robust evidence for treatments to mitigate or avoid weight gain among people being commenced on clozapine. Two studies explored the role of metformin to attenuate weight gain on people commenced on olanzapine, an antipsychotic similar to, clozapine, but with a lower propensity for weight gain. One study showed amelioration of weight gain ${ }^{28}$ while the other reported equivocal results. ${ }^{29}$ To date, no RCTs have examined the effect of concomitant prescription of metformin with clozapine to attenuate weight gain.

Identifying potential biomarkers that predict poor metabolic outcomes can aid in developing personalised medicine, with an aim of using genetic testing to identify those at highest risk of weight gain associated with clozapine, and those who may benefit most from adjunctive metformin. A review by our group identified genetic associations between clozapine, and BMI and metabolic syndrome, in genes including LEP, HTR2C and rs381328. ${ }^{30}$ Another meta-analysis of people with T2DM identified that rs11212617 was associated with better glycaemic response to metformin. ${ }^{31}$

The primary aim of this study is to investigate the effectiveness of metformin in attenuating weight gain in people with schizophrenia newly commenced on clozapine. We hypothesise that people who are co-commenced on metformin will have significantly lower end point weight, compared with those started on placebo. We also aim to investigate secondary outcomes including comparative rate of conversion to T2DM, proportion with $>5 \%$ gain in body weight, derangement of metabolic syndrome components, change in diet and appetite and association with genetic biomarkers of change in weight among trial participants.

\section{METHODS AND ANALYSIS}

\section{Study design/setting}

The CoMET study is a 24-week parallel, double-blind, placebo-controlled, randomised controlled trial (RCT) testing the efficacy of adjunctive metformin to attenuate weight gain in clozapine-naïve people with schizophrenia or schizoaffective disorder who are newly commenced on clozapine. We aim to recruit 86 participants with diagnoses of schizophrenia or schizoaffective disorder within 2 weeks of being commenced on clozapine.

Participants will be randomised to receive treatment as usual including clozapine plus either metformin or placebo. The dose of metformin will be titrated over a 
3-week period up to $2 \mathrm{~g}$ /day, as tolerated. Placebo dosing will be increased accordingly.

The study will be conducted across four Hospital and Health Service (HHS) Districts in South East Queensland: Metro North HHS, Metro South HHS, West Moreton HHS and Gold Coast HHS. Participants will be recruited with support of treating clinicians from inpatient units, clozapine clinics, community care units and community clinics.

\section{Study population}

The CoMET study will recruit 86 participants with schizophrenia or schizoaffective disorder who have commenced treatment with clozapine in the last 2 weeks. Participants will have a BMI between 18 and $40 \mathrm{~kg} / \mathrm{m}^{2}$. Participants will be excluded from the study if they have pre-existing diagnosis of T2DM, or are already taking metformin or any other weight-lowering medications. The full inclusion and exclusion criteria are detailed in online supplementary appendix 1.

\section{Patient screening and enrolment}

Clozapine is a highly monitored drug in Australia. Individuals must undergo a medical screening process prior to clozapine commencement, and once commenced on clozapine, they need to attend structured weekly medical appointments, with weekly biometric measurements and blood tests for the first 18 weeks of clozapine treatment. Thereafter, patients are reviewed 4 weekly as per existing clozapine protocol. For people newly commenced on clozapine in Australia, preregistration with a clozapine manufacturer patient monitoring system is required.

This preregistration requires the involvement of the hospital service clozapine coordinator and/or mental health pharmacist. Hence, the participating hospital service clozapine coordinator and pharmacists will be aware of all people being commenced on clozapine. The study team will liaise with the clozapine coordinators and mental health pharmacists to identify potential study participants. Potential participants who agree to being approached by the research team will be provided with written and verbal information about the study and invited to consider participation.

The study screening process will begin by assessing the capacity of all potential participants. Once potential participants are deemed to have capacity, they will be thoroughly informed about the trial's components and requirements. If they wish to proceed, informed consent will be obtained and witnessed.

Previous research by members of our group found that in Queensland, approximately eight people are newly commenced on clozapine per year per 100000 catchment population. ${ }^{32}$ The participating HHS cover a population of at least 2.5 million people, with an estimated 200 patients commenced on clozapine annually. With a conservatively estimated $30 \%$ study participation rate, 60 people could be commenced in the study annually.
Figure 1 documents the flow of participants from screening to follow-up.

\section{Allocation concealment, randomisation and masking}

Participants will be randomised once written consent has been obtained and the study screening assessments have determined that the participant is eligible. Participants will be randomised to metformin (active treatment) or placebo in a 1:1 ratio using blocks of 4 via a computer-generated randomisation table. The treating team, participants and the research team will all be blinded to allocation of intervention. Randomisation will not be stratified by site. The randomisation list will be generated by an independent statistician not directly involved in the delivery of intervention or outcome assessment. The randomisation list will be provided to an independent pharmacy team at the Princess Alexandra Hospital. This pharmacy team will be the only service with the ability to unblind patients. Participants will be provided with a 24 hours contact number in case there is an emergent situation where it is crucial that medical staff know whether they are receiving metformin or placebo.

Allocation concealment will be maintained by using placebo tablets that are identical in packaging, appearance, colour and taste to the metformin tablets and by increasing the number of placebo tablets to match the titration of metformin. All other study assessments and procedures will be identical between the two groups.

\section{Treatment protocol}

\section{Metformin group}

Those in the metformin group will be provided with an extended release (XR) formulation of metformin with their evening meal for 24 weeks. Metformin XR $500 \mathrm{mg}$ tablets will be used. To reduce potential side effects metformin will be titrated as tolerated over a 3-week period with $500 \mathrm{mg}$ XR daily given the first week, $1000 \mathrm{mg}$ XR daily the second week and $2000 \mathrm{mg}$ XR daily for the remainder of the study. The titration regime will be discussed weekly with the co-ordinating principal investigator or delegate and the study endocrinologist or delegate. If $2000 \mathrm{mg}$ XR daily is not tolerated than participants will be given the maximum tolerated dose.

Participants will also receive treatment as usual. In keeping with Queensland Health standards of care for psychosis this may include individualised combinations of psychopharmacology, behavioural interventions, dietary advice, physical activity programmes, rehabilitation and associated clinical services. Participant's engagement in dietary and physical activity programmes will be recorded in the case files.

\section{Placebo group}

Those in the placebo group will be provided with a daily dose of placebo with their evening meal for 24 weeks. The placebo tablets are identical to the metformin XR tablets. The dose will also be titrated as tolerated over 3 weeks with one tablet being given in the first week, then two 


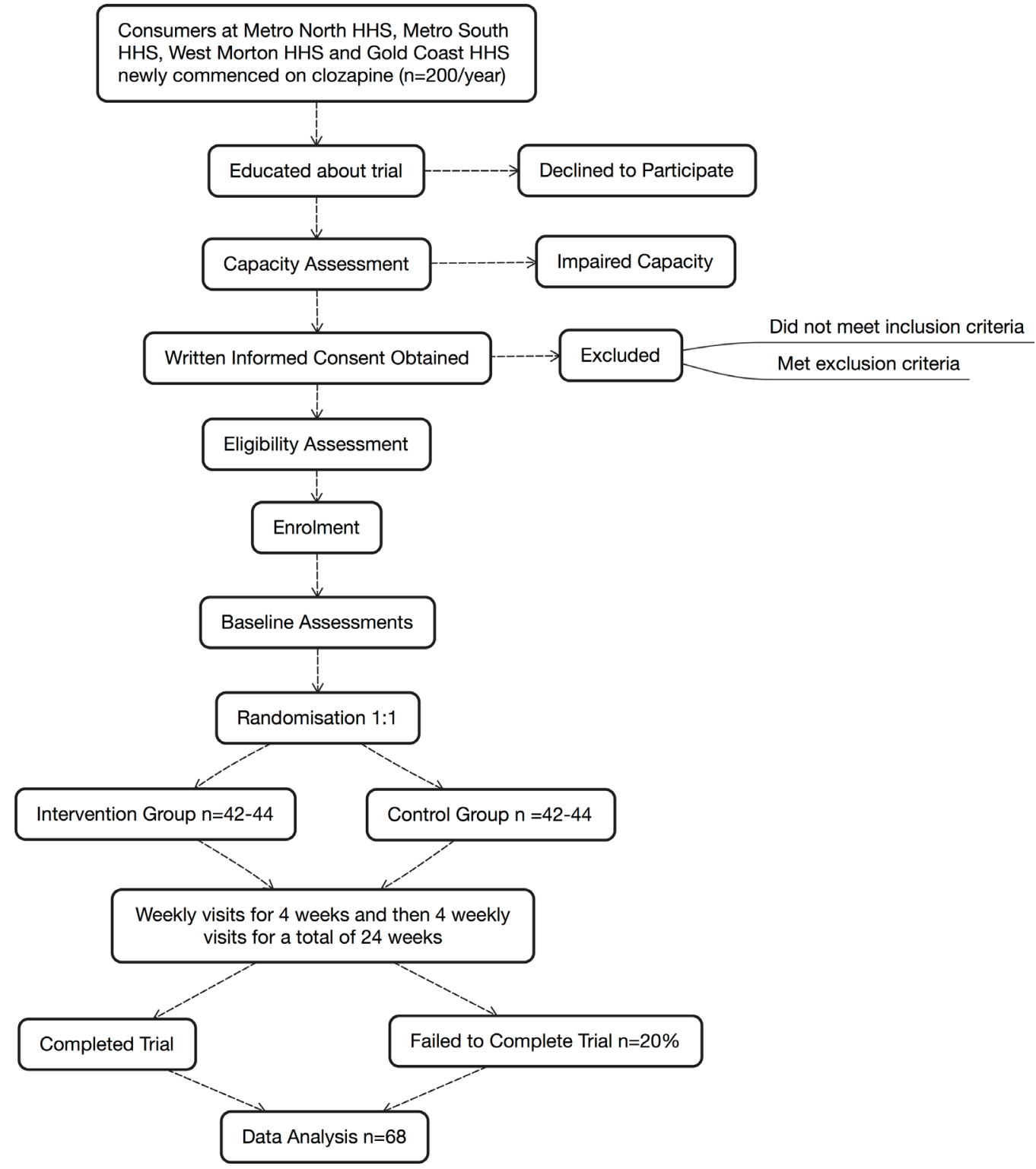

Figure 1 Flow chart of the CoMET trial. HHS, Hospital and Health Service.

tablets from week 2 and then four tablets from week 3. If four tablets are not tolerated then participants will be given the maximum tolerated dose. Those in the placebo group will also receive treatment as usual.

Adherence will be monitored through return of unused tablets and tablet counts at each visit.

\section{Dose justification}

In a recent meta-analysis, the mean metformin dose used in RCTs comparing metformin to placebo in people without T2DM who were prescribed clozapine ranged from 250 to $1500 \mathrm{mg} .{ }^{19}$ Clinical recommendations for the use of metformin in T2DM suggest starting at $500 \mathrm{mg}$ and titrating up to $2000 \mathrm{mg}$ based on serial blood glucose measurements. ${ }^{33} 34$ A study by Chiu et $a l^{35}$ compared metformin doses of 500 and $1000 \mathrm{mg}$ among people already obese on clozapine. They found a statistically significant reduction in body weight after 12 weeks in the $1000 \mathrm{mg}$ group but not the $500 \mathrm{mg}$ group. This suggests that a dose of at least $1000 \mathrm{mg}$ is required for consistent weight reduction in patients on clozapine. We have elected to use the maximum tolerated dose within the recommended dosing range of metformin XR (500$2000 \mathrm{mg}$ ) to explore the maximum possible effect with metformin.

\section{Outcomes}

Primary

The primary outcome will be end point body weight in $\mathrm{kg}$ at 24 weeks, between the placebo and metformin groups.

\section{Secondary}

Secondary outcome measures are:

- Rate of conversion to T2DM (fasting 2 hours glucose tolerance test and haemoglobin A1c);

- Metabolic syndrome components ${ }^{36}$ (waist circumference, fasting glucose, HDL, total cholesterol, triglycerides and blood pressure); 
- Homeostatic model assessment of insulin resistance and secretion based on fasting glucose and insulin;

- Diet and appetite (Food Craving Inventory);

- Physical activity (International Physical Activity Questionnaire (IPAQ) and Simple Physical Activity Questionnaire (SIMPAQ));

- Proportion with weight gain of $5 \%$ or more at end point versus baseline;

- Dropout rates;

- Quality of life (Assessment of Quality of Life (AQoL)). A range of symptom, cognitive and plasma drug measures will also be examined to explore whether any group differences in end point weight can be attributed to differences in the following clinical assessments:

- Psychotic symptoms (Positive and Negative Syndrome Scale (PANSS));

- Psychosocial Function (Global Assessment of Functioning (GAF));

- Cognitive function (Brief Cognitive Assessment Tool for Schizophrenia (B-CATS), Test of Premorbid Functioning (TOPF) and California Verbal Learning Test (CVLT-II);

- Clozapine/norclozapine levels and ratio.

\section{Tertiary}

Collect DNA for future study into genetic biomarkers associated with weight gain with clozapine and/or response to metformin.

\section{Trial visits, assessments and outcome measures}

Study visits and assessments, identical in both groups, will be conducted as per the schedule mentioned in table 1 . Study visits will be weekly for the first 4 weeks and then every 4 weeks for the remainder of the study. The investigational product will be dispensed at every study visit. Physical measurements and adverse drug reaction monitoring will be conducted by the research team at every study visit. A range of validated clinical assessments (table 1 ), will be conducted at weeks 4, 8, 12, 16, 20 and 24. Participants will have three blood tests during the study, at baseline and weeks 12 and 24. Every effort will be made to ensure that these blood tests coincide with mandatory blood tests for clozapine monitoring.

People with pre-existing T2DM, chronic kidney disease and pregnancy will be excluded (see online supplementary appendix 1). Preclozapine investigations will be ordered by the treating team, as part of the mandatory workup for clozapine, including fasting serum glucose, estimated glomerular filtrate rate and beta-human chorionic gonadotropin. These data will be used for the screening of participants. Vitamin $\mathrm{B}_{12}$ serum levels will be monitored at baseline, week 12 and week 24 as a rare side effect of metformin includes vitamin $\mathrm{B}_{12}$ deficiency.

Blood will also be collected at baseline for future DNA analysis. Participation in this part of the study is optional and separate consent will be sought.

All assessments will be conducted by trained members of the research team. In addition to the scheduled study visits, participants will be contacted regularly by the research trial team during the trial in an effort to improve adherence to the investigational product and increase retention rate. We will record use and dose of concomitant psychotropic medications.

All anthropometric measurements will be collected by the research trial team while participants wear light clothing, after the participants have emptied their bladder and removed their shoes. Height will be recorded at the screening assessment. At each visit, weight will be recorded to the nearest $0.1 \mathrm{~kg}$ using calibrated scales. Waist circumference will be measured in the horizontal plane to the nearest $0.5 \mathrm{~cm}$ using a non-stretchable measuring tape placed around the abdomen at a level halfway between the top of the iliac crest and the bottom of the ribs. ${ }^{37}$ Hip circumference will be measured at the maximum circumference of the buttocks. ${ }^{37}$ The hip/waist ratio is the ratio of hip circumference and waist circumference.

Pulse and blood pressure will be recorded after sitting for 5 min. ${ }^{38}$ Blood pressure will also be recorded in the standing position after the participant has been standing for $2 \mathrm{~min}$.

\section{Positive and Negative Syndrome Scale}

The PANSS, a validated 30-item investigator-rated measure, will be used to measure positive and negative symptoms of schizophrenia. ${ }^{39}$

\section{Global Assessment of Functioning scale}

The GAF is a validated investigator-rated scale incorporating symptom severity, psychological, social, and occupational functioning on a scale from 0 to $100 .^{40}$

\section{IPAQ and SIMPAQ}

The IPAQ is a validated participant recall-based measure of physical activity in the past week. ${ }^{41}$ The SIMPAQ is a participant recall-based measure of physical activity in the past week that is specifically designed for people living with mental illness. ${ }^{42}$

\section{Assessment of Quality of Life}

The AQoL is a validated instrument that measures five health dimensions: illness, independent living, social relationships, physical senses and psychological well-being, and can be used for economic evaluations. ${ }^{43}$

\section{Cognitive assessments}

\section{Test of Premorbid Functioning}

The TOPF is a revised version of the Wechsler Test of Adult Reading and is a measure of premorbid cognitive and memory functioning. ${ }^{44}$

\section{CVLT-II short form}

The CVLT-II short form is a validated test of verbal learning and memory. ${ }^{45}$

\section{Brief Cognitive Assessment}

The B-CATS includes the digit symbol substitution test, trail making test and verbal fluency test. These tests, 
Open Access

Table 1 Schedule of visits and assessments

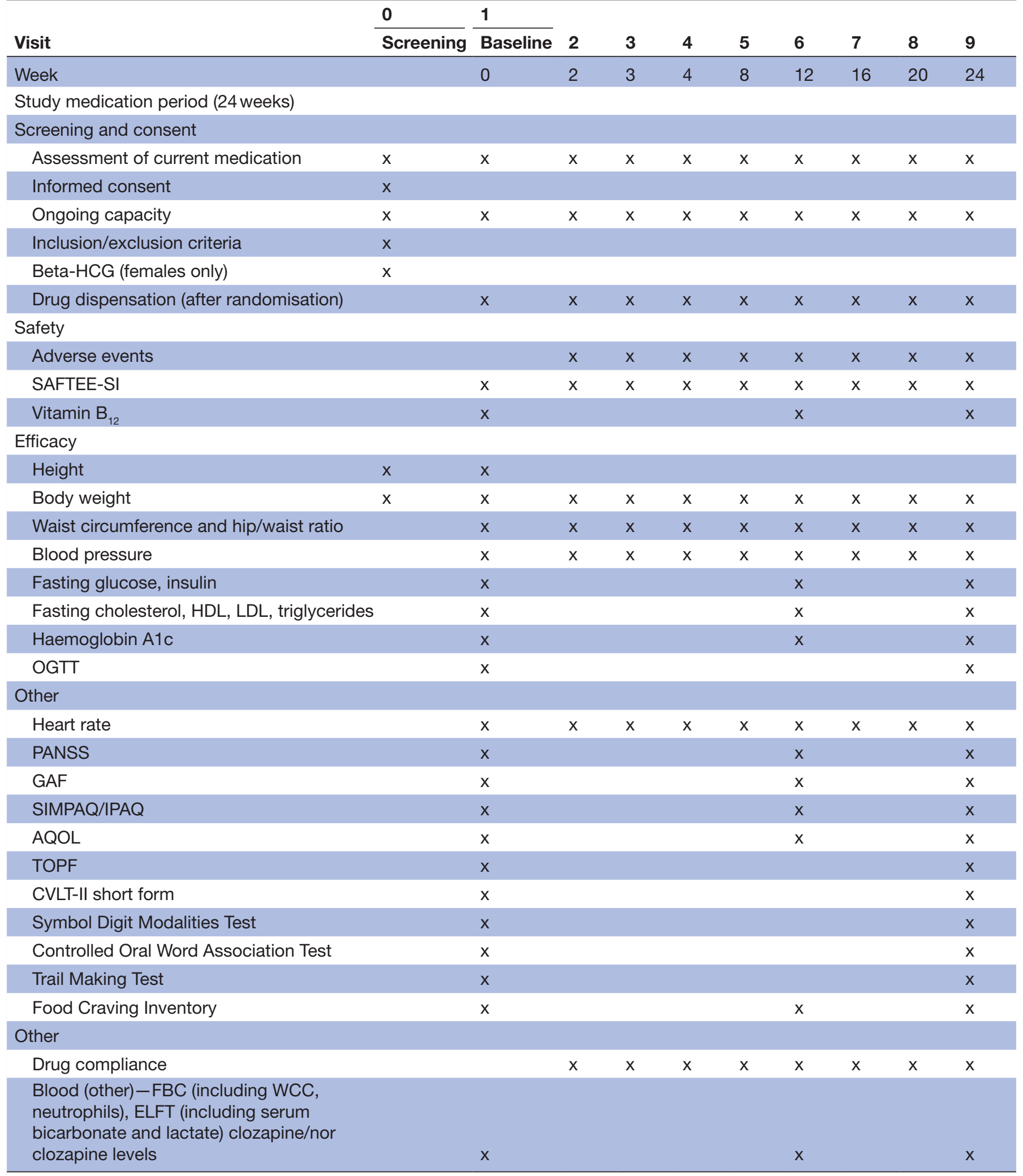

AQoL, Assessment of Quality of Life; CVLT-II, The California Verbal Learning Test second edition; ELFT, Electrolytes and Liver Function Tests; FBC, full blood count; GAF, Global Assessment of Functioning; HCG, human chorionic gonadotropin; HDL, high-density lipoprotein; IPAQ, International Physical Activity Questionnaire; LDL, low-density lipoprotein; OGTT, Oral Glucose Tolerance Test; PANSS, Positive and Negative Syndrome Scale; SAFTEE-SI, Systematic Assessment for Treatment Emergent Events-Systematic Inquiry; SIMPAQ, Simple Physical Activity Questionnaire; TOPF, Test of Premorbid Functioning. 
respectively, complex processing speed, visual attention and task switching and semantic fluency and strategy generation. The B-CATS is validated and has good reliability and consistency and can be delivered in around 10 min. $^{46}$

\section{Food Craving Inventory}

The Food Craving Inventory is a validated measure of food cravings, and is based on participant self-report. It has two scales, one for subjective cravings and the other for consumption of particular foods. ${ }^{47}$

Data will be initially recorded on paper case report forms. Data will be checked by two independent members of the research team and then entered into an electronic data management software programme (RedCAP). All confidential data will be securely stored as per Good Clinical Practice guidelines.

\section{Statistical methods \\ Sample size}

We powered our study based on the primary outcome, body weight at 24 weeks, using the repeated measures analysis of covariance approach. Sample size was estimated using data from a meta-analysis of metformin for clozapine-associated obesity conducted by our group. ${ }^{19}$ To observe a minimal clinically difference in weight change of $3.12 \mathrm{~kg}$, assuming SD of 9.6 in both groups (overall SD from the meta-analysis), $\alpha=0.05$ and correlation between baseline and repeated measures of 0.7 , we will require 34 participants per group to achieve $80 \%$ power. Allowing for an attrition rate of $20 \%$ from baseline to follow-up, we will need to recruit 86 participants across the four sites.

\section{Data analysis}

Analysis will be conducted according to intention-totreat principle with participants analysed in the group they were originally allocated to regardless of treatment compliance. Baseline characteristics will be summarised using mean and SD for continuous variables, and n (\%) for categorical variables. The distribution of continuous variables, if skewed, where appropriate will be transformed using log-transformation. Baseline characteristics between the two groups will be compared using either the t-test (continuous data) or $\mathrm{X}^{2}$ test/Fisher's exact test (categorical data). The primary outcome, end point weight, will be analysed using a mixed model repeated measure model (MMRM). The MMRM is a superior approach in controlling for type I error and minimise bias as it does not impute or exclude participants with missing data. ${ }^{48}$ We will include weight at baseline assessment, intervention group, visit and visit by intervention in the model. We will also test the sensitivity of our results by imputing for missing values in the primary outcome using multiple imputation. We will test for the impact of potential confounders, such as concomitant medications, on the results and adjust for these as appropriate. Results will be presented as mean difference along with $95 \%$ CIs. Secondary outcomes will be analysed in a similar fashion using MMRM for normal outcomes or generalised linear mixed models for non-normal outcomes. Number needed to treat and number needed to harm will be calculated. All analyses will be conducted using SAS software, V.9.4. ${ }^{49}$

\section{Participant safety}

Metformin has been used extensively for the treatment of T2DM. It is well tolerated, with hypoglycaemic episodes very rare unless combined with other antihyperglycaemics. ${ }^{24}$ There are no known pharmacokinetic interactions between metformin and clozapine. Previous studies exploring the tolerability of metformin in people with schizophrenia taking various antipsychotics found that the reported side effects were very similar between the metformin and placebo groups. ${ }^{50}$

The most common side effect of metformin is gastrointestinal disturbance, which includes diarrhoea, flatus, nausea, abdominal discomfort and reduced appetite. ${ }^{33}$ Metformin-associated gastrointestinal disturbance normally resolves in the first few weeks and can be reduced by using the XR formulation, taking metformin with the evening meal and titrating the dose slowly. ${ }^{33}$

Other possible rarer side effects include taste disturbance, vitamin $\mathrm{B}_{12}$ deficiency, lactic acidosis and hepatobiliary disorders. Serious side effects including vitamin $\mathrm{B}_{12}$ deficiency, lactic acidosis and hepatobiliary disorders are rare. Most cases of lactic acidosis have occurred in patients with diabetes with significant renal failure and other risk factors. ${ }^{33}$ We will monitor for the side effects by testing vitamin $B_{12}$ levels, electrolytes and liver function at baseline, week 12 and week 24 .

The use of iodinated contrast materials concomitantly with metformin may be associated with nephropathy. Our participants' kidney function will be protected during the study by withholding metformin or placebo for 48 hours after intravenous contrast is administered. ${ }^{33}$

Metformin is relatively safe even when taken in overdose. Despite ingestion of large amounts of metformin (up to $85 \mathrm{~g}$ ) hypoglycaemia has not been observed. ${ }^{33}$ Lactic acidosis has been reported as a consequence of overdose in people with pre-existing T2DM. ${ }^{33}$ Pre-existing T2DM is an exclusion criteria, and participants who develop T2DM during the study will be withdrawn. Any dose taken above the highest recommended daily dose of $2000 \mathrm{mg}$ will be considered an overdose and recorded and reported as a serious adverse event (SAE). To reduce the risk of a serious overdose, the investigational product will be dispensed weekly for the first 4 weeks and then every 4 weeks for the remainder of the study.

Participants will be assessed for possible adverse effects (AEs), at every study visit, including use of the Systematic Assessment for Treatment Emergent Events-Systematic Inquiry (SAFTEE-SI) tool. ${ }^{51} \mathrm{AE}$ can refer to serious and non-SAEs. For this study, an AE is defined as any unfavourable and unintended sign (including abnormal laboratory findings), symptom or disease (new or exacerbated) temporally associated with the use of a medicinal product, whether or not considered related to the 
medicinal product. Medical and surgical procedures will not be classed as AEs. SAEs, as defined in online supplementary appendix 2 , will be reported to the study monitor as soon as possible and to the reviewing ethics committee. Any serious, unexpected adverse events that are causally related to the investigational product will be reported to the Australian Therapeutic Goods Administration and other appropriate regulatory as per the applicable guidelines.

Safety outcomes will also be collected to assess the preliminary safety and tolerability of metformin. These include:

- Number of dropouts between intervention and control arm;

- Number of adverse drug reactions in the intervention and control arm;

- Scores from a structured qualitative interview with participants about their experiences with study drug using the SAFTEE-SI;

- Serum bicarbonate and lactate to assess for lactic acidosis;

- Vitamin $\mathrm{B}_{12}$ levels to assess for vitamin $\mathrm{B}_{12}$ deficiency.

\section{Study completion and withdrawal}

Participants will be deemed to have completed the trial when they complete 24 weeks of dosing. Participants have the right to remove their consent and withdraw from the study at any time and this will be clearly discussed during the screening process. Participants who cease clozapine during the study period will be withdrawn from the study. In addition, participants may also be withdrawn by the investigator if they meet withdrawal criteria (see online supplementary appendix 3).

Any participant withdrawn from the study will have their last observation carried forward in an intention-totreat analysis.

\section{Reimbursement}

Participants will receive honorarium in the form of gift cards to the total value of US $\$ 140$, over the course of the study.

\section{Ethics and dissemination}

The study will be carried out according to the Declaration of Helsinki, the NHMRC National Statement on the Ethical Conduct in Research involving Humans (2007) and the Notes for Guidance on Good Clinical Practice as adopted by the Australian Therapeutic Goods Administration (2000) (CPMP/ICH/135/95) and the ICH GCP Guidelines.

Ethics approval was granted by the Metro South Human Research Ethics Committee HREC/17/QPAH/538 - SSA/17/QPAH/565.

This study has Therapeutic Good Administration Clinical Trial Notification (2017-CTN-02935), and has been listed on the Australian and New Zealand Clinical Trials Registry (ACTRN12617001547336).
An independent Data Safety Monitoring Board will monitor safety data during the trial.

This study protocol has been devised in line with the Standard Protocol Items: Recommendations for Interventional Trials (SPIRIT) guidelines ${ }^{52}$ (see supplemental SPIRIT Checklist).

On study completion, results will be disseminated by peer-reviewed publications and conference presentations, regardless of the findings. Manuscripts will be prepared in accordance with the Consolidated Standards of Reporting Trials 2010 Statement. ${ }^{53}$ Our findings will also be summarised in several brochures, including one designed for feedback to participants and hospital sites that participate in the study.

\section{DISCUSSION}

Clozapine is the most effective antipsychotic for treatment refractory schizophrenia but has the worst metabolic profile of all antipsychotics. Although there is evidence that some pharmacological interventions such as exenatide $^{54}$ and metformin ${ }^{19}$ can reduce weight for people who are already obese taking clozapine, the magnitude of this reduction in weight gain is small. There is a critical need for evidence-based therapies to avert the initial clozapine-associated weight gain. To date, there have been no RCTs examining the effectiveness of metformin to attenuate weight gain in patients newly commenced on clozapine.

This study will examine whether metformin can attenuate weight gain over a 24-week period when compared with placebo as its primary outcome. It will also examine whether metformin has an effect on the components of the metabolic syndrome, rates of conversion to T2DM and changes to diet and exercise.

There are limitations inherent to randomised, placebo-controlled trials, which apply here. The study population who enrol in this trial may not be generalisable to all people commencing on clozapine, nor can the findings for a trial of people commencing clozapine be translatable to other antipsychotic medications.

Reducing the metabolic burden of clozapine from commencement has the potential to reduce the risk of developing cardiovascular disease and T2DM. If efficacious and safe, metformin would be a relatively accessible, cost-effective intervention. Because weight gain is a major concern among people on clozapine, attenuation of weight gain can enhance self-image, adherence and is thus likely to improve health outcomes and the quality of life of people living with schizophrenia. Ultimately, interventions that ameliorate weight gain in those with schizophrenia may also reduce the unacceptable high mortality and morbidity gaps between people living with schizophrenia and the general population.

\section{Author affiliations}

${ }^{1}$ University of Queensland School of Medicine, Brisbane, Queensland, Australia ${ }^{2}$ Metro South Addiction and Mental Health Service, Brisbane, Queensland, Australia 
${ }^{3}$ Queensland Centre for Mental Health Research, Brisbane, Queensland, Australia ${ }^{4}$ Sunshine Coast Health and Hospital Service, Brisbane, Queensland, Australia ${ }^{5}$ Department of Endocrinology, Princess Alexandra Hospital, Brisbane, Queensland, Australia

${ }^{6}$ Queensland Brain Institute, University of Queensland, St Lucia, Queensland, Australia

${ }^{7}$ National Centre for Register-Based Research, Aarhus University, Aarhus, Denmark ${ }^{8}$ Metro North Mental Health Service, Brisbane, Queensland, Australia

${ }^{9}$ West Moreton Health and Hospital Service, Brisbane, Queensland, Australia

${ }^{10}$ Gold Coast Health and Hospital Service, Gold Coast, Queensland, Australia

${ }^{11}$ Pharmacy Department, Princess Alexandra Hospital, Brisbane, Queensland, Australia

${ }^{12}$ University of Queensland School of Pharmacy, Brisbane, Queensland, Australia

Contributors DS, AR, AB and JJMG conceived the study. All authors (DS, NF, AR, JJMG, CL, SP, DF, TS, VM, SSa, SSu, SK, KW, AB) contributed to the study design and planning. NF prepared the first draft of the manuscript with $D S, A R, A B$ and JJMG reviewing and amending early draft versions. KW provided advice on study drug pharmacokinetics and pharmacodynamics. CL provided statistical advice. The site PIs (DS, SP, DF, TS, VM, SSa, SSu and SK) assisted with guiding the protocol drafting in light of local site issues. All authors (DS, NF, AR, JJMG, CL, SP, DF, TS, VM, SSa, $\mathrm{SSu}, \mathrm{SK}, \mathrm{KW}, \mathrm{AB}$ ) edited and contributed to the final version of the manuscript, and all authors gave final approval to the submitted version. For the clinical trial itself, DS is the study chief investigator, AB is the trial coordinator and DS, SP, DF, TS, VM, SSa, SSu and SK are site principal investigators and will be actively involved in participant recruitment. KW will provide pharmacological support and liaison with the dispensing pharmacy, AR will provide endocrinology guidance during the course of the clinical trial. CL will develop the statistical analysis plan, with support from DS, SK and JJMG.

Funding The trial is funded in part from a NHMRC ECF APP1111136 awarded to DS, and a Royal Brisbane and Women's Hospital Project Grant awarded to DF, SP, DS and VM.

Competing interests AR has received speaker honoraria and travel grants from AstraZeneca, Boehringer Ingelheim, Eli Lilly, MSD, Novo Nordisk and Sanofi and has participated on advisory panels for MSD and Novo Nordisk.

Patient consent Not required.

Ethics approval Ethics approval was granted by the Metro South Human Research Ethics Committee HREC/17/QPAH/538-SSA/17/QPAH/565.

Provenance and peer review Not commissioned; externally peer reviewed.

Open Access This is an Open Access article distributed in accordance with the Creative Commons Attribution Non Commercial (CC BY-NC 4.0) license, which permits others to distribute, remix, adapt, build upon this work non-commercially, and license their derivative works on different terms, provided the original work is properly cited and the use is non-commercial. See: http://creativecommons.org/ licenses/by-nc/4.0/

(C) Article author(s) (or their employer(s) unless otherwise stated in the text of the article) 2018. All rights reserved. No commercial use is permitted unless otherwise expressly granted.

\section{REFERENCES}

1. Lawrence D, Hancock KJ, Kisely S. The gap in life expectancy from preventable physical illness in psychiatric patients in Western Australia: retrospective analysis of population based registers. BMJ 2013;346:f2539.

2. Ryan MC, Collins $P$, Thakore $\mathrm{JH}$. Impaired fasting glucose tolerance in first-episode, drug-naive patients with schizophrenia. Am J Psychiatry 2003;160:284-9.

3. Rosenbaum S, Tiedemann A, Sherrington C, et al. Physical activity interventions for people with mental illness: a systematic review and meta-analysis. J Clin Psychiatry 2014;75:964-74.

4. Dipasquale S, Pariante CM, Dazzan P, et al. The dietary pattern of patients with schizophrenia: a systematic review. J Psychiatr Res 2013;47:197-207.

5. Mitchell AJ, Vancampfort D, Sweers K, et al. Prevalence of metabolic syndrome and metabolic abnormalities in schizophrenia and related disorders--a systematic review and meta-analysis. Schizophr Bull 2013;39:306-18.
6. NCCfM H. Psychosis and Schizophrenia in Adults: Treatment and Management: Updated Edition 2014. Psychosis and Schizophrenia in Adults 2014

7. Agid O, Arenovich T, Sajeev G, et al. An algorithm-based approach to first-episode schizophrenia: response rates over 3 prospective antipsychotic trials with a retrospective data analysis. J Clin Psychiatry 2011;72:1439-44.

8. Howes OD, McCutcheon R, Agid O, et al. Treatment-Resistant Schizophrenia: Treatment Response and Resistance in Psychosis (TRRIP) Working Group Consensus Guidelines on Diagnosis and Terminology. Am J Psychiatry 2017;174:216-29.

9. Siskind D, McCartney L, Goldschlager R, et al. Clozapine v. firstand second-generation antipsychotics in treatment-refractory schizophrenia: systematic review and meta-analysis. $\mathrm{Br} J$ Psychiatry 2016;209:385-92.

10. Land R, Siskind D, McArdle P, et al. The impact of clozapine on hospital use: a systematic review and meta-analysis. Acta Psychiatr Scand 2017;135:296-309.

11. Siskind DJ, Harris M, Phillipou A, et al. Clozapine users in Australia: their characteristics and experiences of care based on data from the 2010 National Survey of High Impact Psychosis. Epidemiol Psychiatr Sci 2017;26:1-13.

12. Henderson DC, Nguyen DD, Copeland PM, et al. Clozapine, diabetes mellitus, hyperlipidemia, and cardiovascular risks and mortality: results of a 10-year naturalistic study. J Clin Psychiatry 2005;66:1116-21.

13. Faulkner $\mathrm{G}$, Cohn $\mathrm{T}$, Remington $\mathrm{G}$, et al. Body mass index, waist circumference and quality of life in individuals with schizophrenia. Schizophr Res 2007;90:174-8.

14. Young SJ, Praskova A, Hayward N, et al. Attending to physical health in mental health services in Australia: a qualitative study of service users' experiences and expectations. Health Soc Care Community 2017;25:602-11.

15. Cooper SJ, Reynolds GP, Barnes T, et al. With expert co-authors (in alphabetical order):. BAP guidelines on the management of weight gain, metabolic disturbances and cardiovascular risk associated with psychosis and antipsychotic drug treatment. J Psychopharmacol 2016;30:717-48.

16. , Di Angelantonio E, Bhupathiraju S, et a/Global BMI Mortality Collaboration. Body-mass index and all-cause mortality: individualparticipant-data meta-analysis of 239 prospective studies in four continents. Lancet 2016;388:776-86.

17. Flegal KM, Kit BK, Orpana $\mathrm{H}$, et al. Association of all-cause mortality with overweight and obesity using standard body mass index categories: a systematic review and meta-analysis. JAMA 2013;309:71-82.

18. Strassnig M, Caceda R, Newcomer J, et al. Cognitive deficits, obesity and disability in schizophrenia. Trans/ Neurosci 2012;3:345-54.

19. Siskind DJ, Leung J, Russell AW, et al. Metformin for Clozapine Associated Obesity: A Systematic Review and Meta-Analysis. PLoS One 2016;11:e0156208.

20. Kirpichnikov D, McFarlane SI, Sowers JR. Metformin: an update. Ann Intern Med 2002;137:25-33.

21. Salpeter SR, Buckley NS, Kahn JA, et al. Meta-analysis: metformin treatment in persons at risk for diabetes mellitus. Am J Med 2008;121:149-57.

22. Knowler WC, Barrett-Connor E, Fowler SE, et al. Diabetes Prevention Program Research Group. Reduction in the incidence of type 2 diabetes with lifestyle intervention or metformin. $N$ Engl J Med 2002;346:393-403.

23. Anon. Effect of intensive blood-glucose control with metformin on complications in overweight patients with type 2 diabetes (UKPDS 34). UK Prospective Diabetes Study (UKPDS) Group. Lancet 1998;352:854-65.

24. Bodmer M, Meier C, Krähenbühl S, et al. Metformin, sulfonylureas, or other antidiabetes drugs and the risk of lactic acidosis or hypoglycemia: a nested case-control analysis. Diabetes Care 2008;31:2086-91.

25. Mannucci E, Ognibene A, Cremasco F, et al. Effect of metformin on glucagon-like peptide 1 (GLP-1) and leptin levels in obese nondiabetic subjects. Diabetes Care 2001;24:489-94.

26. Holst JJ. The physiology of glucagon-like peptide 1. Physiol Rev 2007;87:1409-39.

27. Mayfield K, Siskind D, Winckel K, et al. Glucagon-like peptide-1 agonists combating clozapine-associated obesity and diabetes. J Psychopharmacol 2016;30:227-36.

28. Wu RR, Zhao JP, Guo XF, et al. Metformin addition attenuates olanzapine-induced weight gain in drug-naive first-episode schizophrenia patients: a double-blind, placebo-controlled study. Am J Psychiatry 2008;165:352-8. 
29. Baptista T, Martínez J, Lacruz A, et al. Metformin for prevention of weight gain and insulin resistance with olanzapine: a double-blind placebo-controlled trial. Can J Psychiatry 2006;51:192-6.

30. Suetani RJ, Siskind D, Reichhold H, et al. Genetic variants impacting metabolic outcomes among people on clozapine: a systematic review and meta-analysis. Psychopharmacology. In Press. 2017;234:2989-3008.

31. Zhou K, Bellenguez C, Spencer CC, et al. Common variants near ATM are associated with glycemic response to metformin in type 2 diabetes. Nat Genet 2011;43:117-20.

32. Forrester T, Siskind D, Winckel K, et al. Increasing Clozapine Dispensing Trends in Queensland, Australia 2004-2013. Pharmacopsychiatry 2015;48:164-9.

33. Therapuetic Goods Administration. Product Information Apo Metformin XR, Product and Consumer Medicine Information.

34. Desilets AR, Dhakal-Karki S, Dunican KC. Role of metformin for weight management in patients without type 2 diabetes. Ann Pharmacother 2008;42:817-26.

35. Chiu CC, Lu ML, Huang MC, et al. Effects of Low Dose Metformin on Metabolic Traits in Clozapine-Treated Schizophrenia Patients: An Exploratory Twelve-Week Randomized, Double-Blind, PlaceboControlled Study. PLoS One 2016;11:e0168347.

36. Alberti K, Eckel R, Grundy S, et al. Harmonizing the metabolic syndrome. A joint interim statement of the IDF Task Force on Epidemiology and Prevention; NHL and Blood Institute; AHA; WHF; IAS; and IA for the Study of Obesity. Circulation 2009;120:1640-5.

37. Organization WH. Waist circumference and waist-hip ratio: Report of a WHO expert consultation. Geneva: WHO, 2011.

38. Pickering TG, Hall JE, Appel LJ, et al. Recommendations for blood pressure measurement in humans and experimental animals. Circulation 2005;111:697-716.

39. Kay SR, Fiszbein A, Opler LA. The positive and negative syndrome scale (PANSS) for schizophrenia. Schizophr Bull 1987;13:261-76.

40. Jones SH, Thornicroft G, Coffey M, et al. A brief mental health outcome scale-reliability and validity of the Global Assessment of Functioning (GAF). Br J Psychiatry 1995;166:654-9.

41. Craig CL, Marshall AL, Sjöström M, et al. International physical activity questionnaire: 12 -country reliability and validity. Med Sci Sports Exerc 2003;35:1381-95.
42. Rosenbaum S, Ward PB. International Working Group. The Simple Physical Activity Questionnaire. Lancet Psychiatry 2016;3:e1.

43. Hawthorne G, Richardson J, Osborne R. The Assessment of Quality of Life (AQoL) instrument: a psychometric measure of health-related quality of life. Qual Life Res 1999;8:209-24.

44. Wechsler D. Test of premorbid functioning. UK version (TOPF UK). UK: Pearson Corporation, 2011.

45. Baños JH, Martin RC. In: Delis J, Kramer E, Ober KB, eds. California Verbal Learning Test. San Antonio, TX: The Psychological Corporation, Elsevier, 2002.

46. Hurford IM, Ventura J, Marder SR, et al. A 10-minute measure of global cognition: Validation of the Brief Cognitive Assessment Tool for Schizophrenia (B-CATS). Schizophr Res 2017.

47. Nicholls W, Hulbert-Williams L. British English translation of the Food Craving Inventory (FCl-UK). Appetite 2013;67:37-43.

48. Siddiqui $\mathrm{O}$, Hung HM, O'Neill R. MMRM vs. LOCF: a comprehensive comparison based on simulation study and 25 NDA datasets. $J$ Biopharm Stat 2009;19:227-46.

49. Institute S. The SAS system for Windows. Release 9.4. Cary, NC: Institute S, 2018.

50. Wu RR, Jin H, Gao K, et al. Metformin for treatment of antipsychoticinduced amenorrhea and weight gain in women with first-episode schizophrenia: a double-blind, randomized, placebo-controlled study. Am J Psychiatry 2012;169:813-21.

51. Jacobson AF, Goldstein BJ, Dominguez RA, et al. Interrater agreement and reliability measures of SAFTEE: general inquiry vs. systematic inquiry. Psychopharmacol Bull 1987;23:97-101.

52. Chan A-W, Tetzlaff JM, Altman DG, et al. SPIRIT 2013: new guidance for content of clinical trial protocols. The Lancet 2013;381:91-2.

53. Moher D, Hopewell S, Schulz KF, et al. CONSORT 2010 Explanation and Elaboration: updated guidelines for reporting parallel group randomised trials. J Clin Epidemiol 2010;63:e1-e37.

54. Siskind DJ, Russell AW, Gamble C, et al. Treatment of clozapine-associated obesityanddiabeteswithexenatideinadultswithschizophrenia: Arandomizedcontrolledtrial (CODEX). Diabetes Obes Metab. In Press. 2017. 